\title{
Die Mobilität Römischer Soldaten in Friedenszeiten
}

\author{
Peter Herz
}

1

Einleitung

Warum bewegten sich römische Soldaten während der Kaiserzeit überhaupt von dem Standort fort, an dem sie offiziell stationiert waren? Sicherlich war der häufigste Grund ein kriegerischer Einsatz. Diesen bereits oft traktierten Bereich der soldatischen Mobilität möchte ich mit voller Absicht nicht ansprechen, sondern die militärische Mobilität in Friedenszeiten betrachten. Mit anderen Worten, ich möchte in erster Linie die administrative Routine studieren, da sie uns einen wesentlich besseren Einblick in das Alltagsleben des römischen Militärs und seine Verwaltungsroutine gewährt.

Eine wichtige Bemerkung vorab. Man sollte die römische Armee nicht nur unter dem Aspekt ihrer militärischen Einsatzfähigkeit sehen, sondern auch als eine Anzahl von Menschen, die dem Kaiser unbegrenzt zur Verfügung standen. Denn die römische Armee (Heer und Marine) repräsentierte nicht nur den bewaffneten Arm der römischen Macht, sondern war auch die größte organisierte Gruppe an einsatzfähigen Menschen, deren Arbeitspotential vom Kaiser überall und nach seinem Belieben eingesetzt werden konnte. Dies erscheint etwas weniger überraschend, wenn man sich vor Augen führt, daß es ja auch für senatores und equites Romani keine grundsätzliche Trennung zwischen einer militärischen und einer zivilen Verwendung gab.

Die deutliche Differenzierung zwischen Militär und Zivildienst, die wir unter den Tetrarchen nachweisen können, setzt erst ab der Mitte des dritten Jahrhunderts ein und ist der eigentlichen Kaiserzeit eher fremd. Dabei sollte man auf jeden Fall berücksichtigen, daß die Römer Pragmatiker waren und daher jeweils auf die personellen Ressourcen zurückgriffen, die sie gerade zur Hand hatten.

Wie fließend die Grenzen zwischen dem militärischen und dem eigentlich zivilen bzw. hoheitlichen Aufgabenbereich sein konnte, zeigt ein Zeugnis aus dem Archiv der Babatha. Dabei fungiert ein praefectus equitum (wohl der 
Kommandeur einer ala des Provinzheeres) als Vertreter des römischen Staates, vor dem der Eid auf die Richtigkeit der census-Deklaration abgelegt wurde. ${ }^{1}$

Ich beabsichtige in meinem Beitrag eine kurze Übersicht zu den vielfältigen Möglichkeiten zu geben, wie römische Soldaten und Offiziere im Dienste des Kaisers auch in Friedenszeiten kreuz und quer durch das Imperium Romanum verschoben werden konnten, um so etwas die Vielfältigkeit der römischen Administration zu illustrieren. ${ }^{2}$

Am Ende soll eine Art von 'working model' stehen, das nach Belieben ergänzt oder verändert werden kann. Vor dem Hintergrund eines solchen Modells sollte es dann auch möglich sein, die Anstrengungen besser zu beurteilen, die die Mobilisierung und Verlegung großer Truppenverbände in Kriegszeiten notwendig machten. ${ }^{3}$

Eine Feststellung darf aber schon zu Beginn gemacht werden. Es dürfte kaum möglich sein, ein Modell zu entwerfen, das ohne Unterschied für alle Provinzen gültig ist. Dazu sind die strukturellen Voraussetzungen und auch die Aufgabenstellung in den einzelnen Provinzen zu unterschiedlich gewesen.

Mobilität innerhalb der Heimatprovinz

Unter dem Begriff 'Heimatprovinz' ist die Provinz zu verstehen, in der sich der feste Standort der militärischen Einheit befindet, der der Soldat offiziell zugeteilt wurde und wo sein Name in der Stammrolle (matricula) der Einheit verzeichnet ist.

Dabei haben wir zunächst die Außenstellen des Stammlagers zu berücksichtigen, zu denen die Soldaten ausrückten. In diese Kategorie gehören kleinere, vom Hauptlager administrativ abhängige Lager oder Einrichtungen wie Depots und Ziegeleien. Einige Einheiten wie die cohors IIII Vindelicorum scheinen auf

1 B. Isaac, 'Tax collection in Roman Arabia. New evidence from the Babatha archive,' Mediterranean Historical Review 9 (1994), 256-266 = The Near East under Roman rule. Selected Papers (Leiden, New York, Köln 1998), 322-333.

2 Wichtig: P. Faure, 'Combattre ou ne pas combattre: métier légionnaire et mobilité militaire dans la première moitié du III e siècle apr. J.-C.', in C. Wolff, Hg., Le métier de soldat dans le monde romaine. Actes du cinquième congrès de Lyon organisé les 23-25 septembre 2010 par l'universitéJean Moulin Lyon 3 (Lyon 2012), 369-416.

3 Wie weitgespannt Mobilität in der Antike war, beweisen die Beiträge in E. Olshausen und V. Sauer, Hg., Mobilität in den Kulturen der antiken Mittelmeerwelt. Stuttgarter Kolloquium zur Historischen Geographie des Altertums 11, 2011. Geographica Historica 31 (Stuttgart 2014). 


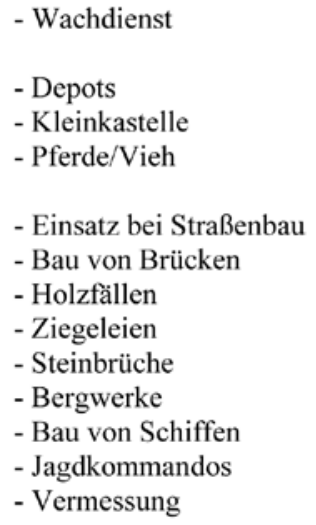

Officium des Statthalters

Schreibstube

- beneficiarii

- eq. /ped. singulares

- Stratores

- Officia von procuratores in der Provinz

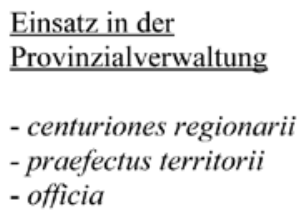

SCHEMA 5.1 Mobilität innerhalb der Heimatprovinz.

den Betrieb von Ziegeleien spezialisiert gewesen zu sein, da sich ihr Name auf vielen Ziegeln nachweisen läßt, die überall in der provincia Germania superior gefunden wurden. ${ }^{4}$ Während diese Einheit wohl in Heddernheim ihr Stammlager hatte, scheinen Baueinheiten überall in der Provinz Ziegeleien betrieben zu haben, die dann in den benachbarten Kastellen verwendet wurden.

Danach kommen Sonderaufträge. In diese Gruppe von Aufträgen gehören die Beschaffung von Holz, Holzkohle und Kalk, die Arbeit in Steinbrüchen und der anschließende Transport der Steine zum Bauplatz sowie der Einsatz beim Bau von Straßen und Brücken. ${ }^{5}$ Kleinere Gruppen von Soldaten dürften auch

4 Vgl. die knappen Angaben bei J. Spaul, Cohors². The Evidence for and a Short History of the Auxiliary Infantry Units of the Imperial Roman Army (Oxford 2000), $290 \mathrm{f}$.

5 Vgl. die Beiträge von Ch. Schmidt Heidenreich, 'Les soldats bâtisseurs dans les camps sous le Haut-Empire', und G. Wesch-Klein, 'Lapidarii des exercitus Romanus', in C. Wolff, Hg., Le métier de soldat dans le monde romaine. Actes du cinquième congrès de Lyon organisé les 23-25 septembre 2010 par l'Université Jean Moulin Lyon 3 (Lyon 2012), 327-344 bzw. 345-365. Am besten sind wir für die großen Steinbrüche in Ägypten informiert, vgl. neben der älteren 
bei der Vermessung eingesetzt gewesen sein. ${ }^{6}$ Eher dem zivilen Sektor zugeordnet sind Hilfsdienste bei der Durchführung des census und der Eintreibung von Sachabgaben der Bevölkerung innerhalb der Provinz.

Als Sondergruppe verstehe ich den Einsatz bei militärischen Jagdkommandos, um etwa wilde Tiere für die venationes zu fangen. Solche Kommandos sind für die Bärenjagd in Germanien, sowie für die Jagd auf Büffel bzw. Wisente auf dem Balkan gesichert. ${ }^{7}$ Damit haben wir wohl nur die Spitze des Eisbergs vor uns, denn der Bedarf an solchen Tieren dürfte enorm gewesen sein. So sind vergleichbare Einsätze etwa bei der Jagd auf Löwen, möglicherweise sogar auf Elephanten in Nordafrika durchaus denkbar.

Eine weitere Gruppe von Aufgaben bedeutete die langfristige oder sogar permanente Abkommandierung zu officia innerhalb der Provinz. Diese Soldaten verblieben zwar noch offiziell auf der Stammrolle ihrer Einheit, waren aber nicht mehr für den Routinedienst oder militärische Kommandos greifbar.

Unabhängig von der Größe einer Provinz oder der Größe des jeweiligen Provinzheeres bestand im Stab eines Statthalters ein ständiger Bedarf vor allem an schreibkundigen Soldaten für das officium. Hinzu kommen die beneficiarii, die equites und pedites singulares und auch die stratores. ${ }^{8}$ Bei den stratores

Arbeit von M.J. Klein, Untersuchungen zu den kaiserlichen Steinbrüchen am Mons Porphyrites und Mons Claudianus in der östlichen Wüste Ägyptens (Bonn 1988); auch D. Peacock und V. Maxfield, Hg., The Roman Imperial Quarries. Survey and Excavation at Mons Porphyrites, 1994-1998. Vol.II. The Excavations (London 2007).

6 Vgl. den Briefwechsel zwischen Plinius und Trajan (Plin. Ep. 10.17 b. und 18) wegen der Delegierung eines Vermessungsspezialisten für Prusa. Der klassische Fall ist der Bau der Wasserleitung von Saldae ( $C I L$ 8. $2728=I L S$ 5795). In der Regel konzentriert man sich auf die Arbeit des veteranus Varius Clemens, übersieht aber, daß beim Bau auch milites classiarii eingesetzt wurden. D. Kienast, Untersuchungen zu den Kriegsflotten der römischen Kaiserzeit (Bonn 1966), 99 Anm. 65 vermutet abkommandierte Soldaten des in Caesarea stationierten Geschwaders.

7 Im Jahre 147 organisierte ein tribunus der cohors I Cilicum zusammen mit vexillationes der legio I Italica, der legio XI Claudia sowie der classis Moesiaca eine große Jagd auf Bären und Wisente (ursis et visontibus prospere captis). Vgl. dazu V. Velkov, G. Alexandrov, 'Venatio Caesariana. Eine neue Inschrift aus Montana (Moesia inferior)', Chiron 18 (1988), 271-277. J. Kolendo, "Des boeufs sauvages" - Les bisons d'Europe dans les balkans depuis l'invasion de Xerxés jusqu'aux invasions slaves', in S. Rucinski, C. Balbuza und Ch. Królczyk, Hg., Studia Lesco Mrozewicz ab amicis et discipulis dedicata (Poznan 2011), 173-180. Für die Involvierung von Soldaten in den Bereich der venationes vgl. auch CIL 6. $130=I L S 2091$ mit Soldaten der cohortes praetoriae, die als venatores immunes und custos vivarii fungieren.

8 Literatur zu diesen Gruppen: J. Ott, Die Benefiziarier. Untersuchungen zu ihrer Stellung innerhalb der Rangordnung des römischen Heeres und zu ihrer Funktion (Stuttgart 1995); J. NelisClément, Militaires et administration au service de l'Empire (1er s. av. J.C.-VI s. ap. J.C.) (Les 
scheint sich ein System abzuzeichnen, bei denen die centuriones stratorum mit Absicht aus legiones genommen wurden, die nicht in der Provinz stationiert waren, wo sie Dienst taten. ${ }^{9}$

Analog dazu bestand auch ein permanenter Bedarf an Soldaten für die officia der unterschiedlichen procuratores. Im Falle der Provinz Germania superior bedeutete dies z.B. die Abkommandierungen zum procurator duarum Germaniarum et Belgicae, daneben sind auch noch Abkommandierungen zum Stab der procuratores der res privata und des patrimonium für diese drei Provinzen zu berücksichtigen. ${ }^{10}$

Vom obergermanischen Limes ist der Einsatz von centuriones der Legionen in kleineren Lagern bekannt, die dort das Kommando hatten, obwohl sie immer noch in der Stammrolle ihrer Einheit geführt wurden. Dazu ein kleines Beispiel:11

Fortunae Conservatrici C(aius) Iul(ius) Maianus (centurio) leg(ionis) VIII Aug(ustae) v(otum) s(olvit) l(ibens) m(erito)

Der bewahrenden Fortuna. Gaius Iulius Maianus, Hauptmann der legio VIII Augusta, hat sein Gelübde gern und wie es sich gebührt eingelöst.

Aus derselben Kategorie wäre auch die Abkommandierung als centurio regionarius bzw. praefectus gentis zu berücksichtigen, wobei dem centurio auch noch einige Soldaten für sein officium zugeordnet waren. ${ }^{12}$

Beneficiarii) (Bordeaux 200o); M.P. Speidel, Guards of the Roman Armies. An Essay of the Singulares of the Provinces (Bonn 1978).

9 Vgl. $A E$ 1985, 829 für L. Valerius Valerianus, dessen centurio strator von der legio VI Ferrata kam. Vgl. M.P. Speidel, 'Valerius Valerianus in charge of Septimius Severus' Mesopotamian campaign', Classical Philology 8o (1985), 321-326 = Roman Army Studies II (Stuttgart 1992), 218-223. Ähnliches scheint sich auch bei Claudius Candidus und Fabius Cilo anzudeuten, deren centuriones stratores von der legio X Gemina (RIT $130=C I L 2^{2} / 14$ Nr. 975) bzw. von der legio VMacedonica (CIL 6.1408 = ILS 1141) kamen.

10 Vgl. H. Bernhard, 'Die römische Geschichte in Rheinland-Pfalz', in H. Cüppers, Hg., Die Römer in Rheinland-Pfalz (Stuttgart 1990. Nachdruck Hamburg 2002), 39-168, bes. 103 für die unterschiedlichen militärischen Gruppen, die in der Administration einer provincia eingesetzt wurden.

11 CIL 13. 7733 (Bad Ems).

12 Vgl. z.B.AE 1990, 858 mit mil(es) leg(ionis) VII Cl(audiae) librarius offici(i) prae(fecti) ter(r)it(orii). Vgl. R. Alston, Soldier and Society in Roman Egypt (London und New York 1995), 86-96 mit Anm.; 113-129 mit Belegen für abkommandierte centuriones bzw. beneficiarii in den Nomen. Ebenso A.Z. Bryen, Violence in Roman Egypt. A Study in Legal Interpretation 
Eine gewisse Vorstellung, zu welchen Kommandos Soldaten innerhalb einer provincia abkommandiert werden konnten, liefern die Papyri. Dabei ist die Gruppe von Quittungen besonders wichtig, die Soldaten bei dem summus curator ihrer Einheit abgeben mußten, wenn man ihnen wegen eines Einsatzes das Heugeld oder das Verpflegungsgeld (epulum) auszahlte, das üblicherweise von ihrer Einheit verwaltet wurde. ${ }^{13}$ Welche konkreten Aufgaben diese Soldaten an ihren Zielorten zu erledigen hatten, wird leider in der Regel nicht angegeben. Es dürften aber vermutlich Aufgaben als Wachsoldaten oder Begleiter von Transporten im staatlichen Auftrag gewesen sein. ${ }^{14}$

Ebenso unklar ist auch die Beteiligung von Soldaten bei wirtschaftlichen Unternehmungen, die im öffentlichen Auftrag innerhalb der Provinz stattfanden. ${ }^{15}$ Hier ist noch ein bedeutender Klärungsbedarf.

\section{Mobilität außerhalb der Heimatprovinz}

Die erste Kategorie enthalt permanente oder längerfristige Abkommandierungen. Hier sind all die Soldaten und Offiziere zu berücksichtigen, die aus einer Militärprovinz in die officia der benachbarten provinciae inermes abkommandiert wurden. Dabei sind sowohl die officia der jeweiligen

(Philadelphia 2013), 19 mit weiterer Literatur in Anm. 15; vgl. dort auch die Texte der Petitionen 214 ff., demnach nahmen regelmäßig centuriones, beneficiarii und stationarii Petitionen aus der Zivilbevölkerung in Empfang. Unklar ist die genaue Funktion des aus C.D. 72.12.4 bekannte centurio. War er ein centurio (regionarius) oder war er lediglich mit der Steuererhebung beschäftigt? Vgl. K. Blouin, Triangular Landscapes. Environment, Society, and the State in the Nile Delta under Roman Rule (Oxford 2014), 267-297 zum Aufstand der boukoloi und der Involvierung der Militärs bei der lokalen Steuereintreibung.

13 R.O. Fink, Roman Military Records on Papyrus (Ann Arbor 1971), Nr. 76 mit einer großen Zahl an Einzelquittungen.

14 Die Bewachung der wichtigen Straßenverbindungen zu den Häfen des Roten Meeres dürfte sehr personalintensiv gewesen sein. Zum Stand der Forschung vgl. H. Cuvigny, Hg., La route de Myos Hormos. L'armée romaine dans le désert oriental d'Égypte. Praesidia du désert de Bérénice I.II (Patis 2003) und dies., Hg., Didymoi. Une garnison romaine dans le désert oriental d'Égypte I. Les fouilles et le materiel (Caire 2012).

15 O. Stoll, 'Armee und Agrarwirtschaft: die "Stationen" vor dem norisch-pannonischen Limes und die Landwirtschaft im "Freien Germanien"', in ders., Römisches Heer und Gesellschaft. Gesammelte Beiträge 1991-1999 (Stuttgart 2001), 452-511 zur möglichen Involvierung der Armee in die Landwirtschaft ihres Stationierungsgebietes. Vgl. auch A. Mócsy, 'Das Problem der militärischen Territorien im Donauraum', Acta Antiqua Academiae Scientiarum Hungaricae 20 (1972), 133-168 = Pannonien und das römische Heer. Ausgewählte Aufsätze (Stuttgart 1992), 125-160. 


\section{Fallweise Kommandos}

Beschaffung von Lebensmitteln,

Kleidung,

Waffen,

Ausrüstung
Officia von Statthaltern

Officia von procuratores

\section{Permanente Kommandos}

Vexillatio von Lyon;

Cohors von Carthago

Einheiten Schwarzmeer;

portus expeditionales; Aufmarschdepots
Offiziere; Mannschaften

- Begleitung von tirones

- Versetzung in andere Provinzheere (Offiziere/Mannschaften)

- Begleitung von Häftlingen zum Gerichtsort bzw. ins Exil oder Haftort

- Versetzung zu cohortes praetoriae, equites singulares, castra peregrina

- Von den castra peregrina

- Außenkommandos

- Sonderaufträge

- Vexillatio der Misenenses/Ravennates

SCHEMA 5.2 Mobilität außerhalb der Provinz.

Provinzstatthalter als auch die officia von diversen procuratores zu berücksichtigen (s.o.).

Die innergallischen Provinzen (Belgica, Lugdunensis, Aquitania) erhielten ihr Personal in erster Linie aus dem Personalbestand der Rheinlegionen. Diese mußten daher neben den officia ihrer jeweiligen Heimatprovinz auch noch die officia von drei Provinzstatthaltern sowie die diverser procuratores bedienen.

Die Zivilprovinzen Dalmatia, Epirus, und Macedonia erhielten ihre Soldaten wahrscheinlich aus dem Mannschaftsbestand der Donauprovinzen. ${ }^{16}$

16 Vgl. R. Haensch, 'Altäre von Armeeangehörigen aus Kleinasien', in A.W. Busch und A. Schäfer, Hg., Römische Weihealtäre im Kontext. Internationale Tagung in Köln vom 3 . Bis zum 5. Dezember 2009. "Weihealtäre in Tempeln und Heiligtümern" (Friedberg 2014), 369-379, bes. $370 \mathrm{ff}$. zu einer offensichtlich permanent stationierten vexillatio nach M.A. Speidel, 'Les longues marches des armées romaines. Réflets épigraphiques de la circulation des militaires dans la province d'Asie au III ${ }^{\mathrm{e}}$ s. ap. J.-C.', Cahiers du Centre Gustave Glotz 20 (2009), 188-210, bes. 206 Anm. 29. Vgl. auch R. Haensch, 'Kolletiones et canalicularii', in C. Wolff, Hg., Le métier de soldat dans le monde romaine. Actes du cinquième congrès de Lyon organisé les 23-25 septembre 2010 par l'UniversitéJean Moulin Lyon 3 (Lyon 2012), 503-511. 
Die kleinasiatischen Provinzen dürften teils von Einheiten an der unteren Donau (westlicher Teil), teils von den Truppen in Kappadokien (östlicher Teil) versorgt worden sein. Bei den kleinasiatischen Binnenprovinzen bezweifle ich, daß die Personalstärke einer dort stationierten cohors quingenaria ausreichend war, um alle dort anfallenden Aufgaben aus dem vorhandenen Personalbestand zu bewältigen.

Eingut bekannter Sonderfall ist dievexillatio dervier germanischen Legionen in Lugdunum, die dort seit Septimius Severus die frühere cohors urbana abgelöst hatte. ${ }^{17}$ Trotzdem ergeben sich hier einige Probleme. Zunächst ist m.E. noch nicht abschließend geklärt worden, wie stark diese vexillatio eigentlich war. War es eine quingenaria oder eine milliaria? Ich würde zu einer vexillatio milliaria tendieren, die verwaltungsmäßig dann auch die Mannschaften in sich vereinigte, die die officia der unterschiedlichen Funktionäre mit Soldaten und Offizieren versorgen mußten, deren Dienstsitz in Lyon war. Da die so entstandenen Mannschaftslücken bei den rheinischen Legionen wahrscheinlich nicht ersetzt wurden, bedeutet dies, daß jede der vier hochkaiserzeitlichen Legionen den Mannschaftsbestand von rund 3 centuriae $=$ ca. 240 Mann oder rund 5 Prozent ihrer Stärke permanent abgeben mußte. ${ }^{18}$

Ebenfalls zur Gruppe der permanenten Abkommandierungen gehört die regelmäßig zum proconsul Africae in Carthago abkommandierte cohors der legio III Augusta. ${ }^{19}$ Hier können wir davon ausgehen, daß diese cohors in einem Rotationsverfahren zwischen Carthago und dem Stammlager ausgetauscht wurde, damit jede cohors (wahrscheinlich abgesehen von der cohors prima) die Chance hatte, dieses begehrte Kommando zu übernehmen. Einen ähnlich permanenten Charakter dürften die vexillationes der niedermösischen Armee auf der Krim und in den verbündeten Städten im Pontus-Gebiet gehabt haben. ${ }^{20}$ Die Stabssoldaten (10 beneficiarii, 2 equites und 1 centurio) des Gavius Secundus, des praefectus orae Ponticae, wurden laut Plinius aus den cohortes

17 The Encyclopedia of the Roman Army (Oxford 2015), 1055 zum Schicksal der cohors XIII urbana von Lugdunum.

18 Ich vermute, daß diese vexillatio von einem equestren tribunus militum kommandiert wurde, der wahrscheinlich alternierend von ein den vier legiones abkommandiert wurde.

19 Y. Le Bohec, La troisième legion Auguste (Paris 1989). Vgl. auch N. Duval, S. Lancel und Y. Le Bohec, 'Études sur la garnison de Carthage. Deux documents nouveaux - Les troupes de Proconsulaire - Le camp de la cohorte urbaine', Bulletin du Comité des Travaux historique (1984), 47-79 = Y. Le Bohec, L'armée romaine en Afrique et en Gaule (Stuttgart 2007), $333-389$.

20 Zum Einstieg: T. Bekker-Nielsen, Hg., Rome and the Black Sea Region. Domination, Romanisation, Resistance. Black Sea Studies 5 (Århus 2006). 
genommen, die in der provincia stationiert waren (ex cohortibus, quibus me praeesse voluisti). ${ }^{21}$

Der ständige Kampf der Amtsträger in den Provinzen um mehr Soldaten für ihre jeweiligen officia wird im Briefwechsel des Plinius besonders deutlich, dürfte aber im Prinzip für alle Provinzen gegolten haben. ${ }^{22}$ In der von Plinius verwalteten Binnenprovinz dürften sich diese Wünsche noch in Grenzen gehalten haben. Wenn man aber die Anzahl der wichtigen Dienststellen etwa in der provincia Aegyptus betrachtet oder in den großen Provinzen wie Britannien, Syrien oder den beiden Germanien, dann dürften die Forderungen dort entsprechend gestiegen sein. ${ }^{23}$

Daneben ist mit längerfristigen Abkommandierungen in wichtige Häfen zu rechnen, um den Nachschub für die Stammeinheit oder das gesamte Provinzheer zu organisieren. Hier wäre zunächst an Aquileia zu denken, wo Untergebene von primi pili der Donaulegionen nachgewiesen sind. ${ }^{24}$ Analog darf man die Existenz von weiteren Häfen mit dieser Aufgabenstellung (portus expeditionales) postulieren. Dazu gehörten wahrscheinlich die Hafenstädte Salona, Thessalonike, Perinthos, Seleucia in Pierien, Laodiceia und Caesarea Maritima.

$\mathrm{Zu}$ den permanenten Kommandos kann man auch den Einsatz als Begleitpersonal zählen, um Rekruten und Nachschub in die Einsatzprovinz zu eskortieren. Bei dem Transfer von Rekruten sind drei unterschiedliche Aufgabentypen zu berücksichtigen.

Erstens, Rekrutierung in den unmittelbaren Nachbarprovinzen. Dies wären die innergallischen Provinzen für den Mannschaftsersatz der Rheinlegionen, möglicherweise auch für den rätischen exercitus. Daneben sind regelmäßige Rekrutierungen in Germania inferior und der Belgica für die britannischen Einheiten anzunehmen.

21 Plin. Ep. 10.21. Die speziellen Beziehungen der provincia Bithynia et Pontus zum exercitus von Moesia inferior scheint sich auch in der alljährlichen Grußgesandtschaft anzudeuten, die die Stadt Byzantion an den dortigen Statthalter schickte (Plin. Ep. 10.43 f.). In Plin. Ep. 10.27 f. wird berichtet, daß der kaiserliche libertus Maximus, der als adiutor des ritterlichen procurator amtierte, mit der Zahl der 10 beneficiarii, die ihm zugeteilt worden waren, nicht zufrieden war und daher zusätzliche Soldaten wünschte.

23 Es wäre wünschenswert, wenn auf der Basis von A. Jördens, Statthalterliche Verwaltung in der römischen Kaiserzeit. Studien zum praefectus Aegypti (Stuttgart 2009) berechnet würde, wieviele Soldaten dort in der Verwaltung eingesetzt waren.

24 L. Mócsy, 'Das Lustrum Primipili und die Annona Militaris', Germania 44 (1966), 312-326, = in ders., Pannonien und das römische Heer. Ausgewählte Aufsätze (Stuttgart 1992), 106120 (mit Nachträgen). Vgl. auch P. Herz, 'Der centurio supernumerarius und die annona militaris', Laverna 10 (1999 [200o]), 165-184. 
Zweitens, Rekrutierungin Provinzen, dievom künftigen Stationierungsgebiet weiter entfernt sind. Ich habe dazu fünf ausgewählte Fälle herangezogen, die sicherlich nur einen Bruchteil der wirklich durchgeführten Rekrutierungsaktionen dieser Art repräsentieren dürften: Rekrutierung in Asia für das Heer in Aegyptus; ${ }^{25}$ Bessi für Mauretania Tingitana; ${ }^{26}$ Thrakische und pannonische Soldaten usw. für die Heere in den beiden germanischen Provinzen; ${ }^{27}$ Cilices für die Donau-Legionen; ${ }^{28}$ Soldaten aus Lykien für das Heer in Syria. ${ }^{29}$

Je nach Größe des Kontingents dürften mehrere Offiziere und eine Anzahl von Mannschaften notwendig gewesen sein, um den Transport zu begleiten und auch die notwendigen administrativen Aufgaben zu erledigen.

Drittens, Transfer von Soldaten zu neuen Einheiten. Hier geht es nicht um die Erfassung und Eskortierung von tirones, sondern um den endgültigen Transfer von bereits ausgebildeten Soldaten. Für diese Soldaten handelt es sich im Gegensatz zu den permanenten vexillationes um eine endgültige Versetzung zu einer neuen Einheit. ${ }^{30}$ Daneben ist bekannt, daß z.B. regelmäßig

$25 \quad$ PSI 1063 (3. September 117 n.Chr.) = Fink 1971, a.a. O. (Anm.13), Nr. 74.

26 M.P. Speidel, 'A thousand Thracian recruits for Mauretania Tingitana', Antiquité Africaine 11 (1977), 167-173, = in ders., Roman Army Studies I (Amsterdam 1984), 341-347. Vgl. auch dens., 'Numerus Syrorum Malvensium. The transfer of a Dacian army unit to Mauretania and its implications', Dacia 17 (1973), 169-177 = Roman Army Papers I (Amsterdam 1984), 149-16o.

27 Hdn. 6.8.2 f. Hierbei wurde ein großes Kontingent von tirones aus dem Balkangebiet unter dem Kommando des späteren Kaisers Maximinus Thrax ausgehoben und nach Germanien verlegt, um dort die Lücken aufzufüllen, die die Einheiten während der expeditio Orientalis erlitten hatten. Vgl. L. Schumacher, Römische Kaiser in Mainz. Im Zeitalter des Principats (27 v.Chr.-284 n.Chr.) (Bochum 1982), 85-92. Auch schon im Jahre 223 ist eine Gruppe von thrakischen Soldaten in Germania inferior nachgewiesen, die offensichtlich zur Auffrischung an den Rhein verlegt worden waren (ILS 2350).

28 M.P. Speidel, 'Contirones et Geta dominus noster', Ziva Antica 39 (1989), 55-56 = AE 1990, 854 = Inscriptions de la Mésie Supérieur, Nr. 3 (Singidunum). Zuletzt D. Grbic, 'The Cilicians in Singidunum. Notes on military epigraphy and topography', Starinar 57 (2007), 221-227.

29 N.P. Milner, 'Athletics, army recruitment and heroisation: L. Sep. Fl. Flavillianus of Oinoanda', Anatolian Studies 61 (2011), 151-167; P. Herz, 'Zur Karriere des Lucius Septimius Flavianus Flavillianus aus Oinoanda', in B. Takmer et al., Hg., Vir doctus anatolicus. Studies in Memory of Sencer Sahin (Istanbul 2016), 464-470 (im Druck).

30 Vgl. den Transfer afrikanischer Soldaten nach Syrien unter Hadrian. M.P. Speidel, Emperor Hadrian's speeches to the African army - A New Text. Monographien des RömischGermanisches Zentralmuseums 65 (Mainz 2006). Für eine Verlegung von syrischen 
ausgewählte Kavalleristen aus den Provinzen zu den equites singulares Augusti abkommandiert wurden. ${ }^{31}$

In eine ähnliche Kategorie müssen wir die Abkommandierung zu den cohortes praetoriae seit Septimius Severus einordnen. Während die im Jahre 193 neu konstituierten cohortes praetoriae in ihrer ersten Welle durch Soldaten und Offiziere formiert wurden, die aus den Beständen der Donau-Legionen abkommandiert worden waren, scheint man später eher zu einer direkten Rekrutierung in ausgewählten Gebieten übergegangen zu sein. Nimmt man die späteren Militärdiplome als Indikator, so scheint sich eine deutliche Bevorzugung des südlichen Balkan anzudeuten. Der Ersatz für die Eliteeinheit der Mauri et Osrhoeni scheint immer aus den Ursprungsgebieten gekommen zu sein.

Fallweise Kommandos betreffen u.a. die Beschaffung und den Transport von Nachschub. Zunächst Kleidung. Aus Hunt's Pridianum wissen wir, daß von dieser Einheit Soldaten abkommandiert worden waren, um Kleidung aus Gallien für Truppen zu begleiten, die in der Provinz Macedonia stationiert waren. ${ }^{32}$ Daneben ist auch die Lieferung von Decken aus Ägypten zum Heer in Kappadokien gesichert. ${ }^{33}$

Hinzu kommen die Waffen. Neben dem Wachpersonal für den Transport zur Einheit ist auch die längerfristige Abkommandierung von Offizieren und Mannschaften in die eigentlichen Produktionsgebiete zu berücksichtigen. Typisch dafür ist die folgende Inschrift: ${ }^{34}$

M. Ulpio Avito (centurioni) leg(ionis) III Aug(ustae) IIII Fl(aviae) opifices loricari(i) qui in Aeduis consist(unt) et vico Briviae Sugnuntiae respondent quiq(ue) sub cura eius fuerunt, erga ibs[os] b(ene) [m]er(enti) pos(uerunt).

Für Ulpius Avitus, dem centurio der legio III Augusta (und) der legio IIII Flavia, die Hersteller von Rüstungen, die sich im Gebiet der Haeduer aufhalten und zu dem vicus Brivia Sugnuntia gehören und die unter seiner

Soldaten nach Nordafrika vgl. Y. Le Bohec, 'Les Syriens dans l'Afrique romaine', Karthago 21 (1987), 81-92 = L'armée romaine en Afrique et en Gaule (Stuttgart 2007), 453-464.

31 M.P. Speidel, Die Denkmäler der Kaiserreiter. Equites singulares Augusti. Beihefte der Bonner Jahrbücher 50 (Bonn 1994).

32 Fink 1971, a.a.O. (Anm. 13), Nr. 63.

$33 B G U$ 7.1564 = Sel. Pap. 2. 395. Zum System der Kleiderbeschaffung (vestis militaris) vgl. J.A. Sheridan, Columbia Papyri IX. The Vestis Militaris Codex (Atlanta 1998), 73-86. 
Aufsicht waren. Sie haben es (für ihn), der sich um sie wohl verdient gemacht hat, errichtet.

Auch bei der Beschaffung von Pferden und Maultieren wurden Soldaten eingesetzt. Administrativ war dies die Aufgabe der centuriones stratores, die aus dem Bestand einer legio in das officium eines Statthalters abkommandiert worden waren. Über welche Distanzen die Pferde für die Einsatzprovinzen herangeschafft wurden, ist ein bisher noch kaum erforschtes Problem.

Daneben waren Soldaten auf mit der Aufsicht bei unterschiedlichen militärischen Baukommandos.befaßt. Dazu gehören die Baumaßnahmen, die nicht aus der Initiative einer Einzelprovinz begonnen wurden, sondern bei denen die entsprechenden Maßnahmen direkt von der Reichszentrale angeordnet wurden. Dazu gehört z.B. der Bau von Schiffen für die expeditio Britannica des Septimius Severus in Mainz. ${ }^{35}$
$\mathrm{I}$ (ovi) $\mathrm{O}$ (ptimo) $\mathrm{M}$ (aximo) Dolicheno pr[o] salute $\mathrm{dd}$ (ominorum) nn(ostrorum) Augg(ustorum) vexill(atio) leg(ionis) P(rimigeniae) p(iae) $\mathrm{f}$ (idelis) agent(ium) in lignar(iis) sub Clod(io) Caerellio (centurione) leg(ionis) I Part(icae) curam agent(e) Maior(io) Urbano optione [A]n[ni] o et Aemil[iano] co(n)s(ulibus).

Dem besten und größten Iuppiter von Doliche. Für das Wohlergehen unserer beiden Herren, der Augusti. Ein Detachement der legio XXII Primigenia, mit den Beinamen 'die gehorsame, die treue', der Männer, die beim Holzfällen eingesetzt sind unter dem Kommando der Clodius Caerellius, eines centurio der legio I Parthica. Aufsicht führte der Feldwebel Maiorius Urbanus. Unter den Konsuln Annius und Aemilianus.

Die übergeordnete Initiative wird durch die Anwesenheit eines centurio ( frumentarius) aus dem Bestand der legio I Parthica aus Mesopotamien gesichert.

DerEinsatz eines centurio in einem Gebiet, das so weit vom Stationierungsort seiner Stammeinheit entfernt war, läßt sich problemlos durch weitere Belege absichern. Denn wenn man die Nachrichten zu den bisher bekannten Verwendungsmöglichkeiten der centuriones sichtet, dann darf man durchaus feststellen, daß die centuriones fast eine Art von 'Allzweckwaffe' des römischen Staates waren. Centuriones stellten nicht nur das organisatorische Rückgrat der 
eigentlichen Armee, sondern sie waren auch vielseitig überall dort einsetzbar, wo es die Autorität des römischen Staates erforderlich machte. ${ }^{36}$ Man könnte sie daher durchaus als eine rangniedere Variante zu den Vertretern der senatorischen und equestren Laufbahn verstehen, die ja in der Praxis auch für alle Aufgaben eingesetzt werden konnten.

Centuriones konnten daher jederzeit alle möglichen Verwaltungsaufgaben oder polizeiliche Funktionen übernehmen. Sie konnten Steuern eintreiben oder Baumaßnahmen aller Art überwachen, wenn diese im Interesse der Zentralregierung notwendig waren. Daher konnten sie auch ohne weiteres zu Einsatzorten versetzt werden, die weit von ihren Stammeinheiten entfernt waren. ${ }^{37}$

Ob die Delegierung eines centurio immer sachlich gerechtfertigt war oder lediglich dazu diente, die Bedeutung eines höheren Funktionärs zu unterstreichen, läßt sich kaum endgültig entscheiden. ${ }^{38}$

\section{$4 \quad$ Einsatz in der innerbetrieblichen Kommunikation}

Am Ende jedes Dienst- bzw. Feldzugsjahres mußte der aktuelle Ist-Bestand einer Einheit zusammengefasst werden, um dann an den Kommandeur des Provinzheeres gemeldet zu werden. Dies dürfte in der Regel durch einen

36 Am besten sind wir zu den centuriones der beiden Heere am Rhein informiert, vgl. O. Richier, Centuriones ad Rhenum. Les centurions légionaires des armées romaines du Rhin (Paris 2004). Materialreich, aber auf die Periode der Severer beschränkt ist die Arbeit von P. Fauré, L'aigle et le cep. Les centurions légionaies dans l'Empire des Sévères (Bordeaux 2013), der 539 ff. einen gut kommentierten Katalog der bekannten centuriones bietet.

37 Vgl. etwa I. Piso und C. Cupcea, 'Ein centurio regionarius der legio X Fretensis in Dakien', Tyche 29 (2014), 115-123 mit weiterem Material. Vgl. auch B. Rankov, 'A contribution to the military and administrative history of Montana', in A.G. Poulter, Hg., Ancient Bulgaria. Papers presented to the International Symposium on the Ancient History and Archaeology of Bulgaria II (Nottingham 1983), 40-73. Ähnlich auch A.M. Hirt, Imperial Mines and Quarries in the Roman World. Organizational Aspects $27 B C-A D 235$ (Oxford und New York 2010), 170 u. 412 Nr. 618 zu einem centurio der legio XXII Primigenia, der offensichtlich in den Steinbrüchen von Karystos eingesetzt war.

38 Vgl. etwa P. Herz, Studien zur römischen Wirtschaftsgesetzgebung. Die Lebensmittelversorgung (Stuttgart 1988), 77 nach Dig. 13.7.43.1 und 119 Anm. 67 nach CIL 14. 125 = ILS 2223 mit einem centurio ann(onae) oder ann(onarius). Vgl. H. Pavis d'Escurac, La préfecture de l'annone. Service administratif imperial d'Auguste à Constantin (Rome 1976), 101 Anm. 74. Ähnlich AE 1977, 171. Man beachte auch den centurio operum in CIL 14. 125 = ILS 2223. Leider läßt sich der Quelle nicht entnehmen, aus welcher Truppe diese beiden centuriones delegiert worden waren. 


\begin{tabular}{l|l|}
\cline { 2 - 2 } $\begin{array}{l}\text { Innerhalb } \\
\text { der Provinz }\end{array}$ & \multicolumn{1}{c|}{$\begin{array}{c}\text { Außerhalb } \\
\text { der Provinz }\end{array}$} \\
$\begin{array}{l}\text { Einheit } \\
\text { Regelmäßige Meldungen an den Statthalter }\end{array}$ & $\begin{array}{l}\text { Statthalter } \\
\text { Regelmäßiger } \\
\text { Nachrichtenverkehr nach } \\
\text { Rom }\end{array}$ \\
$\begin{array}{l}\text { Statthalter } \\
\text { Nachrichten an einzelne oder alle Einheiten des Provinzheeres }\end{array}$ & \\
$\begin{array}{l}\text { Statthalter } \\
\text { Nachrichten an zivile Verwaltung (procuratores) } \\
\begin{array}{l}\text { Provinziallandtage } \\
\text { einzelne Gemeinden }\end{array}\end{array}$ &
\end{tabular}

Von Rom ausgehende Kommunikation betreffend:
a) Politische Ereignisse (Kaiserhaus, Ernennungen usw.)
b) Administrative Veränderungen (Praefectus praetorio, Statthalter)
c) Einsätze und ihre Planung
d) Versetzungen von Offizieren/Mannschaften
e) Rekrutierungen
f) Beschaffungsmaßnahmen
g) Bauvorhaben

SCHEMA 5.3 Einsatz in der offiziellen Kommunikation.

berittenen Kurier erfolgt sein. Der so ermittelte Ist-Bestand des gesamten Provinzheeres wurde dann durch einen speziellen Boten an die Reichszentrale weitergeleitet. Dies dürfte regelmäßig durch berittene Boten bzw. im Falle der provinciae transmarinae per Schiff erfolgt sein. Auf Grund dieser Informationen wurde dann entschieden, welcher Ersatzbedarf an Rekruten, an Waffen und sonstiger Ausrüstung im letzten Jahr entstanden war und jetzt ersetzt werden mußte. Diese Meldungen bildeten z.B. eine administrative Grundlage für den berühmten libellus, den man nach dem Tode des Augustus vorfand und in dem der aktuelle Zustand des römischen Heeres festgehalten wurde. ${ }^{39}$

39 Suet. Aug. 101.4: .. . tertio breviarium totius imperii, quantum militum sub signis ubique esset, quantum pecuniae in aerario et fiscis et vectigaliorum residuis, “. . . im dritten libellus eine kurze Übersicht über das gesamte Imperium, wie viele Soldaten im Dienst waren und wo sie sich befanden, wieviel Geld sich in der Staatskasse und den Sonderkassen befand und was an Steuergeldern noch ausstand", zusammen mit Tac. Ann. 1.11.3 f.:... cum proferri libellum recitarique iussit. Opes publicae continebantur, quantum civium sociorumque in armis, quot classes regna provinciae, tributa aut vectigalia, et necessitates ac largitiones, "Darauf ließ er einen libellus herbeibringen und vorlesen. Darin waren enthalten, wie viele Bürger und Bundesgenossen unter Waffen standen, wie viele Flotten, Königreiche, 
Diese administrative Routine generierte regelmäßig eine Serie von Schreiben, die von Rom aus an die für eine Rekrutierung ausgewählten Provinzen übermittelt werden mußten, damit diese hinsichtlich des Bedarfs und des Zeitpunktes, bis zu dem die tirones bereitgestellt werden mußten, informiert waren.

Parallel dazu ist von einer weiteren Serie an Schreiben auszugehen, die an das MilitärderZielprovinz gerichtet wurde, damit diese das notwendige Personal für die Überführung der Rekruten abkommandieren konnte. ${ }^{40}$ Für diese Personen mußten Begleitschreiben und Berechtigungsschreiben für die vehiculatio ausgestellt und übermittelt werden. Falls zivile Stellen (Provinz(landtag), Städte) involviert waren, die z.B. ziviles Begleitpersonal delegieren mußten, waren auch hier Aufforderungsschreiben notwendig. Es war daneben ein gesondertes Schreiben an den procurator der Rekrutierungsprovinz notwendig, damit dieser das Geld für das viaticum bereitstellte. Im Fall eines Transportes über See mußten zusätzlich Schiffe organisiert werden, was eventuell durch den Einsatz der lokalen corpora der navicularii geschah.

Da der römische Staat über keine eigenständige Transportflotte verfügte, wurden die notwendigen Schiffe über den privaten Sektor beschafft. Beim Transport über See boten die corpora naviculariorum, die in einem engen Vertragsverhältnis zum Staat standen, die geeignete organisatorische Grundlage. ${ }^{41}$ Beim Flußtransport konnte man die notwendigen Transportschiffe sowohl konfiszieren als auch bei den privaten Eigentümern anmieten (System der locatio conductio operum). Während wir dank der besonderen Quellensituation für die provincia Aegyptus vergleichsweise gut informiert sind, fehlen für die anderen Regionen des Reiches die notwendigen zusammenfassenden Studien zum Einsatz von Schiffen. ${ }^{42}$

In ähnlicher Manier dürften auch offizielle Schreiben in die bevorzugten Produktionsgebiete von Waffen und Kleidung gerichtet worden sein, damit diese informiert wurden, daß sie innerhalb eines festgelegten Zeitraums bestimmte Kontingente produzieren bzw. bereitstellen sollten. Auch in diesen

Provinzen, direkte oder indirekte Steuern, dazu Verbindlichkeiten und Schenkungen es gab."

40 Dies war natürlich nur notwendig, wenn die Rekruten nicht direkt von Personal aus dem Rekrutierungsgebiet begleitet wurden.

41 Vgl. P. Herz 1988, a.a.O. (Anm. 38), 90 ff., 120 ff. Daneben auch L. De Salvo, Economia privata e pubblici servizi nell' impero romano. I corpora naviculariorum (Messina 1992).

42 Ausgangspunkt für Ägypten ist die Arbeit von C. Adams, Land Transport in Roman Egypt. A Study of Economics and Administration in a Roman Province (Oxford 2007). 
Fällen waren schriftliche Aufforderungen für die Transportbegleiter und den zuständigen procurator notwendig.

Zusätzlich ist auch die ständige Information der Einheiten zu berücksichtigen, die in einer Provinz stationiert waren. Dies geschah durch Rundschreiben des Statthalters an die ihm nachgeordneten Einheiten. Beispielhaft dafür ist das folgende Zeugnis. ${ }^{43}$

Marius Maximus trib(unis) et praef(ectis) et praepositis $\mathrm{n}$ (umerorum) salutem.

Quid scripsi Minicio Martiali proc(uratori) Aug(ustorum) n(ostrorum) et notum haberetis adplicui. Opto bene valeatis.

\section{Ex(emplum)}

Curae tibi sit et quaesturas n(umerorum) per quos transit Goces legatus Parthorum missus ad d(ominos) n(ostros) fortissimos imp(eratores) / secundum morem xenia ei offere. Quid autem in quoque numero erogaveris scribe mihi.

Gazica / Appadana / Du[r]a / Ed[da]na / Bi[blade ]

Marius Maximus grüßt die Tribune, Praefekten und Führer der Einheiten. Was ich Minicius Martialis, dem procurator unserer Kaiser, geschrieben habe, habe ich angefügt, damit Ihr es zur Kenntnis nehmt. Ich hoffe, daß es euch gut geht.

Kopie: Kümmere dich darum und auch die Kassenverwaltungen der Einheiten, durch die Goces, der von den Parthern geschickte Gesandte, zu unseren Herren, den allertapfersten Kaisern, fährt, daß ihm entsprechend der Sitte Gastgeschenke überreicht werden. Was aber in welcher Einheit ausgegeben wird, schreibe mir.

(Verteiler): Gazica / Appadana / Dura / Eddana / Biblada.

Ein in seinem Umfang nur schwer zu evaluierender Bereich betrifft die Überstellung von Gefangenen in die Provinzhauptstadt bzw. nach Rom. Der bekannteste Fall ist sicherlich der des Apostels Paulus, obwohl solche Überstellungen auch in Märtyrerakten noch sehr oft erwähnt werden. ${ }^{44}$ Es

43 P. Dura 6o B = Fink 1971, a.a.O. (Anm. 13), Nr. 98.

44 Ob Paulus wirklich auf seinem Weg von Jerusalem nach Caesarea von fast einer kompletten cohors begleitet wurde, wie es die Apostelgeschichte (Act.Ap. 23.23: 200 Infanteristen, 70 Reiter, 200 Leichtbewaffnete) berichtet, erscheint mir fraglich. Dies könnte vom Autor der Apostelgeschichte aufgebauscht worden sein, um die Bedeutung des Apostels hervorzuheben. 
genügt an Bischof Ignatius von Antiochia zu erinnern, der zu seiner Hinrichtung von Syrien nach Rom überstellt wurde. ${ }^{45}$ Bei der Begleitmannschaft dürfte es sich um abkommandierte Soldaten bzw. Offiziere gehandelt haben, die dem jeweiligen Provinzheer entnommen wurden und die wahrscheinlich bereits im Stab des Provinzstatthalters anwesend waren. Daneben müssen wir aber auch noch die Variante berücksichtigen, daß die Aufforderung zur Verhaftung und Überstellung einer Person direkt von der Reichszentrale ausging, die dann auch das notwendige Personal mitschickte.

Ähnlich sieht es beim Wachpersonal für die Überstellung von Gefangenen an ihren Exilort (in insulam) aus. Wenn die hochrangigen Gefangenen direkt von Rom aus verschickt wurden, dürfte diese Aufgabe von Soldaten der cohortes praetoriae erledigt worden sein, die möglicherweise auch als Wachpersonal vor Ort blieben. ${ }^{46}$ Dies allerdings nur in den Fällen, in denen nicht die Zielgemeinde als Kollektiv die Verantwortung für die Überwachung der Gefangenen übernahm. Ähnlich sah es auch bei der Überstellung von Personen aus, die zur Zwangsarbeit verurteilt worden waren, also die Gruppe der in metalla damnati und ihrer anschließenden Bewachung. ${ }^{47}$

5 Konklusion

Auf der vorläufigen Basis dieser Informationen darf man vermuten, daß in Friedenszeiten wahrscheinlich regelmäßig 25 bis 30 Prozent der nominellen Mannschaftsstärke zumindest der Legionen ständig anderweitig eingesetzt waren und daher nur bedingt für militärische Einsätze zur Verfügung standen.

Um sich eine solche Aussage zu verdeutlichen, kann man von einem idealen Zustand der Einsatzbereitschaft ausgehen, in dem 100 Prozent der Truppe jederzeit verfügbar waren. In einem solchen idealen Falle würden sich alle Soldaten und Offiziere, die nominell zu einer Einheit gehörten, auch wirklich in ihrer Garnison befinden und könnten daher nach einer minimalen Mobilisierungsphase in voller Einsatzstärke ausrücken.

45 Vgl. W.H.C. Frend, Martyrdom and Persecution in the Early Church (Oxford 1965), 197-201. Für den geistigen Hintergrund vgl. C.R. Moss, The Other Christians. Imitating Jesus in Ancient Christian Ideologies of Martyrdom (Oxford 2010), $41 \mathrm{ff}$. Obwohl nicht ausdrücklich gesagt, dürfte Ignatius wahrscheinlich wie Paulus ein civis Romanus gewesen sein.

46 F. Stini, Plenum exiliis mare. Untersuchungen zum Exil in der römischen Kaiserzeit. Geographica Historica 27 (Stuttgart 2011), geht auf die technischen Details kaum ein.

Hirt 2010, a.a.O. (Anm. 37). 
Dabei ist anzumerken, daß das von Th. Fischer vorgestellte Stärkeschema für eine legio kaum haltbar ist. ${ }^{48}$ In diesem Modell werden zusätzlich zu einer vermuteten Einsatzstärke von 4800 Infanteristen und 120 Kavalleristen völlig arbiträre Zahlen z.B. für Handwerker innerhalb der Truppe (400), Beschäftigte bei den verschiedenen Stäben der Einheit (260) oder Abkommandierte zu anderen Stäben oder Aufgaben (610) angesetzt. Im Gegenteil muß man wohl die Masse dieser Männer von der eigentlichen Kampfstärke abziehen, was die wirkliche Einsatzstärke einer solchen legio natürlich entscheidend verringern mußte.

Ähnliche Überlegungen gelten auch für die Soldaten der auxilia. Wenn man z.B. die bedeutende Zahl an Kleinkastellen entlang des obergermanischraetischen Limes nimmt, dann stellt sich ganz natürlich die Frage nach der Herkunft der dort eingesetzten Soldaten. Wenn man nicht Soldaten irregulärer numeri als Besatzung des Limes (Besatzung der Türme, Streckenposten) vermuten möchte, dann können die am Limes eingesetzten Soldaten an sich nur aus den regulären Einheiten der größeren Kastelle in der Nachbarschaft gekommen sein. Dieser permanente Einsatz im Grenzdienst dürfte die Stammbesatzungen dieser Kastelle und damit auch deren weitere Einsatzfähigkeit deutlich beeinträchtigen haben. In vielen Kastellen dürfte daher nur noch eine Rumpfbesatzung zurückgeblieben sein. ${ }^{49}$

Wahrscheinlich konnte man auf römischer Seite diesem Dilemma zwischen der Sicherung der Grenze und der Bereitschaft für einen größeren Einsatz nur begegnen, wenn man einige Einheiten möglichst aus dem eigentlichen Grenzdienst herausnahm. Ich gehe daher davon aus, daß es wahrscheinlich in jedem Provinzheer eine Reihe von Einheiten aus der Gruppe der auxilia gab, die einem höheren Grad der militärischen Einsatzfähigkeit besaßen und die man in Kriegszeiten bevorzugt für Einsätze heranziehen konnte. Dabei würden die verschiedenen alae auch wegen ihrer schnelleren Einsatzfähigkeit die interessantesten Kandidaten für solche Einheiten liefern. ${ }^{50}$

Einer der Gründe, warum eine so hohe Zahl an römischen Soldaten auch in Friedenszeiten außerhalb der Truppe eingesetzt werden mußte, um die zivile Verwaltung und auch die öffentliche Sicherheit zu gewährleisten, ist sicherlich

48 Th. Fischer, Die Armee der Caesaren. Archäologie und Geschichte (Regensburg 2012), 17 nach H. von Petrikovits, Die Innenbauten römischer Legionslager während der Prinzipatszeit (Opladen 1975).

49 H. Bernhard 1990, a.a.O (Anm. 10), 39-168, bes. 89-93 zu den Besatzungen am Limes.

$50 \quad$ Vgl. etwa M.P. Speidel, 'Exploratores. Mobile elite units of Roman Germany', Epigraphische Studien 13 (1983), 63-78, bes. 78 f. = Roman Army Studies II (Stuttgart 1992), 89-104, bes. 96f. zum Einsatz der exploratores Divitenses in Mauretanien. 
in der Grundkonzeption der römischen Herrschaft zu suchen. Neben der römischen Armee existierte auf überregionaler Ebene praktisch keine bewaffnete Autorität, die in der Lage gewesen wäre, im größeren Umfang polizeiliche Aufgaben zu übernehmen. Daher muß man selbst für Italien, also das Kernland des Imperium Romanum, feststellen, daß es z.B. in Unteritalien Regionen mit einer geminderten staatlichen Autorität gab, in denen der römische Staat erst dann intervenierte, wenn die Unsicherheit für die Autorität des Kaisers unerträglich wurde. ${ }^{51}$

Was daher Aelius Aristides in seiner Lobrede auf die Stadt Rom als positive Errungenschaft der römischen Herrschaft hervorhebt, stimmt sicherlich aus der Perspektive eines reichen und gebildeten Vertreters der griechischen Oberschicht in Kleinasien, geht allerdings an den Problemen des Reiches weit vorbei. $^{52}$

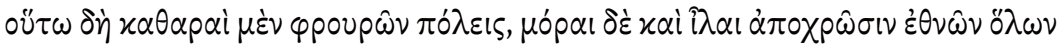

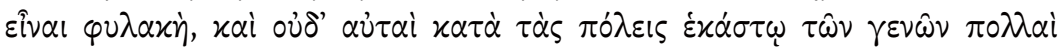

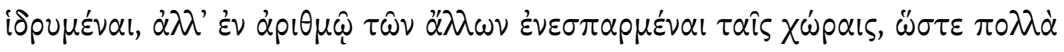

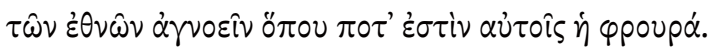

So sind die Städte frei von Besatzungen, Kohorten und Reiterabteilungen genügen zur Beaufsichtigung ganzer Provinzen, und nicht einmal jene sind in größerer Menge auf die Städte der einzelnen Stämme verteilt, sondern sie leben entsprechend der übrigen Bevölkerung verstreut im Land, so daß viele der Provinzen gar nicht wissen, wo ihre Besatzung steht...

Aelius Aristides verstand das Imperium Romanum als ein Reich, in dem Städte und städtisch geprägte lokale Gesellschaften dominierend waren. Er verdrängte damit allerdings die unpassende Erkenntnis, daß die Realität in vielen Regionen des Reiches völlig anders aussah. In diesen Gebieten hatte es das Imperium Romanum bestenfalls in Ansätzen geschafft, eine Struktur zu entwickeln, in denen städtische Gemeinden (und ihre Führungsschichten) einen großen Anteil der Verwaltung übernehmen konnten. Wenn der römische Staat

$5^{1}$ Vgl. M.L. Caldelli, M.F. Petraccia und C. Ricci, 'Praesidia urbis et Italiae. I mestieri della tutela e della sicurezza', in C. Wolff, Hg., Le métier de soldat dans le monde romaine. Actes du cinquième congrès de Lyon organisé les 23-25 septembre 2010 par l'UniversitéJean Moulin Lyon 3 (Lyon 2012), 285-299 zur internen Sicherheit in Italien.

52 Ael. Arist. Orat. 26. 67. Nach R. Klein, Die Romrede des Aelius Aristides, herausgegeben, übersetzt und mit Erläuterungen versehen (Darmstadt 1983). 
also den Anspruch realisieren wollte, in solchen Gebieten seine Herrschaft durchzusetzen, dann war das römische Militär das einzige Machtinstrument, aus das er zurückgreifen konnte. ${ }^{53}$

Es ist das Eingeständnis der traurigen Tatsache, daß die römische Armee an vielen Stellen eingreifen mußte, um die Defizite einer nicht adäquat mit Personal ausgestatteten zivilen Verwaltung auszugleichen, während die eigenen Kräfte kaum ausreichend waren, die militärische Sicherung der Grenzen zu gewährleisten. Kamen dann noch Gebiete wie Iudaea-Palaestina hinzu, in denen sich die römische Herrschaft zusätzlich mit einer internen Opposition konfrontiert sah, dann waren die Probleme fast vorprogrammiert und die militärischen Kapazitäten kamen sehr schnell an ihre Grenzen. ${ }^{54}$

Die in meinem Beitrag vorgestellten Materialien haben die Fragestellung sicherlichnicht erschöpfend behandeln können, sondern sie konnten im besten Falle nur die Grundzüge und auch die potentiellen Erkenntnismöglichkeiten des Themas 'Mobilität des römischen Heeres in Friedenszeiten' aufzeigen. Ich hoffe aber, daß es mir trotz dieser Einschränkungen gelungen ist, die Notwendigkeit und auch den möglichen Ertrag einer ausführlichen Beschäftigung mit einer solchen Fragestellung zu verdeutlichen. Es ist noch viel zu tun!

Regensburg, Silvester 2015

53 Ich greife hier auf einige Überlegungen zurück, die ich an anderer Stelle formuliert habe. Vgl. P. Herz, 'Das Entstehen einer Provinz. Gedanken zum römischen Recht und zur römischen Politik', in U. Lohner-Urban und P. Scherrer, Hg., Der obere Donauraum 50 v.Chr. bis 50 n.Chr. (Berlin 2015), 185-197.

54 Vgl. B. Isaac, The Limits of Empire. The Roman Army in the East (Oxford 1990), 101-160 zur Verteilung der Einheiten und der praesidia. Vgl. auch S.Th. Parker, Romans and Saracens. A History of the Arabian Frontier (Winona Lake 1986) und dens., Hg., The Roman Frontier in Central Jordan. Final Report of the Limes Arabicus Project 1980-1989 I.II (Washington, D.C. 2006). 